\title{
12th Grade Completion, No Diploma
}

National Cancer Institute

\section{Source}

National Cancer Institute. 12th Grade Completion, No Diploma. NCI Thesaurus. Code C67134.

Indicates that a person was in school through 12th grade but they did not receive a high school diploma. 